Portland State University

PDXScholar

Winter 3-13-2019

\title{
Jet Rebound from Hydrophobic Substrates in Microgravity
}

Karl Jeffrey Theodore Cardin

Portland State University

Follow this and additional works at: https://pdxscholar.library.pdx.edu/open_access_etds

Part of the Fluid Dynamics Commons Let us know how access to this document benefits you.

Recommended Citation

Cardin, Karl Jeffrey Theodore, "Jet Rebound from Hydrophobic Substrates in Microgravity" (2019). Dissertations and Theses. Paper 4830.

https://doi.org/10.15760/etd.6706

This Thesis is brought to you for free and open access. It has been accepted for inclusion in Dissertations and Theses by an authorized administrator of PDXScholar. Please contact us if we can make this document more accessible: pdxscholar@pdx.edu. 
Jet Rebound from Hydrophobic Substrates in Microgravity

by

Karl Jeffrey Theodore Cardin

A thesis submitted in partial fulfillment of the requirements for the degree of

\author{
Master of Science \\ in \\ Mechanical Engineering
}

Thesis Committee:

Mark Weislogel, Chair

Raúl Bayoán Cal

Gerald Recktenwald

Portland State University

2019 


\begin{abstract}
We experimentally investigate the phenomena of large jet rebound, a mode of fluid transfer following oblique jet impacts on hydrophobic substrates. We initially seek to describe the jet rebound regimes in tests conducted in the weightless environment of a drop tower. A parametric study reveals the dependence of the flow structure on the relevant dimensionless groups such as Reynolds number and Weber number defined on the velocity component perpendicular to the substrate. We show that significantly larger diameter jets behave similarly as much smaller jets demonstrated during previous terrestrial investigations is some parameter ranges while the flow is fundamentally different in others. Level-set numerical predictions are provided for comparisons where practicable. Simple models are developed predicting landing geometry and the onset of instability that are found to yield good agreement with experiments and simulations. Improving our understanding of such jet rebound opens avenues for unique transport capabilities.
\end{abstract}




\section{Acknowledgments}

I would like to thank my advisor Mark Weislogel for his wisdom, guidance and motivation and for encouraging me to think creatively and independently. I am also grateful to the other members of my thesis committee Raúl Bayoán Cal and Gerald Recktenwald for the support they provided throughout my time in the program. I am thankful to the whole Dryden Drop Tower team who created a unique, fruitful, energetic, and memorable research environment. Financial support for this work was provided in part by NASA Cooperative Agreements NNX16AC38G and NNH15ZTT001N. Finally, I wish to express my gratitude to my family for their unwaivering and enthusiastic support. 


\section{Contents}

Abstract $\quad$ i

Acknowledgments $\quad$ ii

List of Tables $\quad$ V

List of Figures $\quad$ ix

Nomenclature $\quad$ x

1 Introduction 1

2 Theory 5

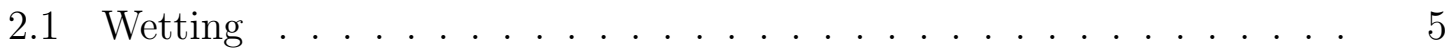

2.2 Landing Flow Retraction . . . . . . . . . . . . . . . . . 7

2.3 Rim Instability . . . . . . . . . . . . . . . . . . . 9 9

2.4 Energy Model . . . . . . . . . . . . . . . . . . 9

2.5 Splashing . . . . . . . . . . . . . . . . . . . . . . 11

3 Methodology 12

3.1 Apparatus ............................ 12

3.2 Hydrophobic Substrate . . . . . . . . . . . . . . . 14

3.3 Data Collection and Reduction . . . . . . . . . . . . . 15 
3.4 Numerical Method . . . . . . . . . . . . . . . . . . . 15

4 Results $\quad 16$

5 Conclusion $\quad 24$

6 Parallel Investigations $\quad 27$

6.1 Hazards of Lid Removal in Microgravity . . . . . . . . . . . . . . . . 27

6.2 Particle Ejection . . . . . . . . . . . . . . . . . . 30

6.3 Capillary Fluidics Demonstration (Water Ping-Pong) . . . . . . . . . 31

$\begin{array}{ll}\text { Bibliography } & 31\end{array}$ 


\section{List of Tables}

3.1 Parameters for a representative selection of drop tower tests. . . . . . 15 


\section{List of Figures}

1.1 Schematic of oblique jet impact with landing flow and rebound from a hydrophobic substrate: (a) top view, (b) profile view, and (c) crosssection view of the landing flow at the point of maximum width $w$ with bounding rims identified by radius $r \ldots \ldots 2$

2.1 Sessile droplets on flat, chemically uniform hydrophillic (a) and hydrophobic (b) substrate. . . . . . . . . . . . . . . 6

2.2 A sessile droplet on a textured superhydrophobic substrate. . . . . . 6

2.3 Time averaged images from drop tower tests of landing flows with the predicted convergence angle $\beta$ overlaid. Tests: (a) $\phi_{i}=15.1^{\circ}, \mathrm{We}_{\perp}=$ 1.50 (D1), (b) $\phi_{i}=18.3^{\circ}, \mathrm{We}_{\perp}=10.05$ (D3), and (c) $\phi_{i}=33.5^{\circ}$, $\mathrm{We}_{\perp}=37.12(\mathrm{D} 4) . \ldots \ldots \ldots \ldots$

3.1 Photograph of the Dryden Drop Tower at Portland State University. . 13

3.2 Drop tower experiment rig with critical items identified. . . . . . . . 14 
4.1 Top view still images taken from drop tower test footage of oblique impacts of a water jet with a superhydrophobic $\left(\theta=158^{\circ}\right)$ substrate in a nearly weightless environment. Regimes include: (a) stable, $\phi_{i}=$ $15.1^{\circ}, \mathrm{We}_{\perp}=1.50(\mathrm{D} 1)$, (b) unstable, $\phi_{i}=18.3^{\circ}, \mathrm{We}_{\perp}=10.05$ (D3), and (c) fishbone, $\phi_{i}=33.5^{\circ}, \mathrm{We}_{\perp}=37.12$ (D4). Light dashed lines indicate the location where the jet leaves the substrate. Dark dashed lines outline the stable rebounded jet profile which does not change with time. Scale bar is $1 \mathrm{~cm} . \ldots \ldots \ldots \ldots$

4.2 Regime map illustrating the observed dependence of flow structures emerging from the oblique impact of a rebounding jet from a superhydrophobic substrate. Marker size is proportional to the diameter of the jet and black bold markers represent numerical simulations. The horizontal line at $\mathrm{Re}=3000$ marks the approximate transition to turbulent flow for the incident jet and the vertical line at $\mathrm{We}_{\perp}=17.5$ marks the predicted onset of splashing behavior (Eq. 2.16 with $C_{1}=1 / 2$. The fit coefficient $C_{1}=1 / 2$ is justified by Fig. 4.4). The shaded region represents the extent of Celestini et al. [7]. . . . . . . . . . . . . . . 18

4.3 Still images of a tangent jet taken from drop tower test footage in (a)1g before the drop and (b) low-g following the drop package release. In 1-g the jet attaches to the substrate and advances as a rivulet (dashed line). In low-g the growing varicose perturbations deflect the jet away from the substrate (dashed curved segments). The downstream jet in (b) is not in contact with the substrate. . . . . . . . . . . 
4.4 Normalized landing flow width $w / d_{j}$ as a function of $\mathrm{We}_{\perp}$. Gray line indicates a maximum normalized landing flow width of $w / d_{j} \sim 2$ for stable rebounds. Marker size is proportional to $d_{j}$ and black bold markers represent simulations. . . . . . . . . . . . . . .

4.5 (a) Top and profile views of time-averaged composite image and (b) sketch of the landing region for the double rivulet jet collision regime (D5). The landing flow film splits producing a third 'terminal rim'. The two edge rivulets rebound from the substrate at the point of intersection with the terminal rim only to collide and coalesce downstream forming a single rebounding jet. The triangular void in the flow observed from above is outlined with a dashed line in (a). . . . . . .

4.6 Still images taken from drop tower test footage of a normal jet impingement in 1-g (a) before the drop and low-g (b) after the drop package release. In $1-g$ the fluid leaving the downstream edge of the landing flow feeds a puddle of height $\sim 2 l_{c}$. In low-g a low Weber number 'jet' is formed which may exhibit dripping behavior, emitting large droplets, and producing a large attached blob. Important features of the fluid free surface are outlined. . . . . . . . . . . . . . . .

4.7 Profile and top view comparisons of numerics with drop tower tests for $d_{j}=6 \mathrm{~mm}$ jet. Comparisons include: (a) stable, $\phi_{i}=15.1^{\circ}, \mathrm{We}_{\perp}=1.50$ (D1), (b) unstable, $\phi_{i}=18.3^{\circ}, \mathrm{We}_{\perp}=10.05$ (D3), and (c) fishbone, $\phi_{i}=33.5^{\circ}, \mathrm{We}_{\perp}=37.12(\mathrm{D} 4) . \ldots \ldots \ldots$ 
5.1 Images from drop tower tests showing the impact of a liquid jet with a concave hydrophobic substrate. Flow rate increases from left to right increasing the intact length of the rivulet. The curvature of the substrate suppresses the rebound of the jet. Dashed lines show the rivulet intact length. . . . . . . . . . . . . . . . .

6.1 (a) A diagram of the experimental setup,(b) an image sequence from lid removal drop tower test showing $\sim 0.5 \mathrm{~s}$ to $2.1 \mathrm{~s}$, and (c) a detail view of the satellite droplets that are generated. . . . . . . . .

6.2 Schematic of particle ejection experiment for (a) a buoyant sphere and (b) triangular prism resting on the bottom of the reservoir. (c) Drop tower test image sequence showing the ejection of a $\sim 40 \mathrm{~mm}$ diameter hydrophobic sphere. . . . . . . . . . . . . . . .

6.3 (a) ESA astronaut Tim Peake demonstrating the ping-pong paddles to approximately 300 school children during a live stream, (b) NASA astronaut Scott Kelly demonstrating the ping-pong paddles on his 100th day in space, (c) and a time sequence of a droplet bouncing between paddles. . . . . . . . . . . . . . . . . . . . 


\section{Nomenclature}

\section{Latin Letters}

\begin{tabular}{|c|c|}
\hline$d_{j}$ & jet diameter \\
\hline$E$ & energy \\
\hline$E_{K E}$ & kinetic energy \\
\hline$E_{S}$ & surface energy \\
\hline$f$ & material fraction of substrate \\
\hline$g_{o}$ & gravitational acceleration at sea level \\
\hline$h$ & landing flow thickness \\
\hline$H$ & model landing flow thickness \\
\hline$l_{c}$ & capillary length \\
\hline$l$ & landing flow length \\
\hline$L$ & model landing flow length \\
\hline$P$ & momentum \\
\hline$r$ & rim radius \\
\hline$r_{s}$ & substrate roughness ratio \\
\hline$v$ & jet velocity \\
\hline$v_{p}$ & capillary pinching velocity \\
\hline$v_{r}$ & rim retraction velocity \\
\hline$v_{\|}$ & parallel jet velocity \\
\hline$v_{\perp}$ & perpendicular jet velocity \\
\hline
\end{tabular}




$$
\begin{array}{ll}
w & \text { maximum landing flow width } \\
W & \text { model landing flow width }
\end{array}
$$

\section{Greek Letters}

$\begin{array}{ll}\beta & \text { rim convergence angle } \\ \theta & \text { Young contact angle } \\ \theta^{*} & \text { apparent contact angle } \\ \mu & \text { dynamic viscosity } \\ \rho & \text { density } \\ \sigma & \text { surface tension } \\ \tau_{p} & \text { capillary pinching time } \\ \tau_{r} & \text { landing flow residence time } \\ \phi_{i} & \text { impact angle } \\ \phi_{r} & \text { rebound angle }\end{array}$

\section{Dimensionless Groups}

Bo $\quad$ Bond number

$\mathrm{Bo}_{a} \quad$ Bond number based on impact acceleration

Re Reynolds number

We Weber number

$\mathrm{We}_{\perp} \quad$ perpendicular Weber number

$\mathrm{We}_{p} \quad$ Weber number based on jet radial perturbation velocity

\section{Subscripts}

$\perp \quad$ perpendicular to the substrate 
variable pertaining to capillary pinching 


\section{Chapter 1}

\section{Introduction}

Liquid jet impingement on solid substrates is a thoroughly studied field due to the beauty, variety, and applicability of the phenomena. The thermophysical properties of the fluid and nature of the solid substrate determines a vast array of outcomes from smooth radial films and hydraulic jumps for wetting substrates, to crowning and splashing for non-wetting liquids. Jet impact on non-wetting substrates has remained relatively unstudied when compared to wetting cases. This investigation focuses on the flow structures resulting from oblique water jet impact with a superhydrophobic surface in the low-gravity (low-g) environment of a drop tower where capillary and inertial forces dominate.

With reference to Fig. 1.1, when an oblique water jet impacts a sufficiently hydrophobic substrate the radial landing flow extension is limited by surface tension. Fluid accumulates along the landing flow edge creating relatively thick bounding rims, Fig. 1.1(c). As the jet-substrate impact angle $\phi_{i}$, Fig. 1.1(b), decreases, the streamwise advection associated with the jet velocity component parallel to the substrate $v_{\|}$ stretches the landing flow downstream resulting in the leaf-shaped geometry observed in Fig. 1.1(a). The rims collide at the downstream apex giving rise to a rebounded jet that leaves the substrate due to vertically imbalanced forces. The non-wetting conditions of the substrate are critical to the dynamics of the process. 
(a)

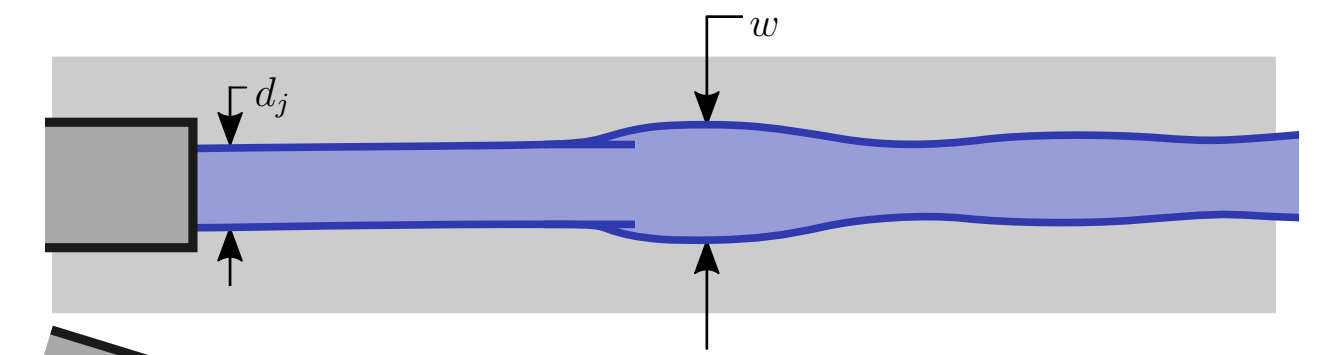

(b)

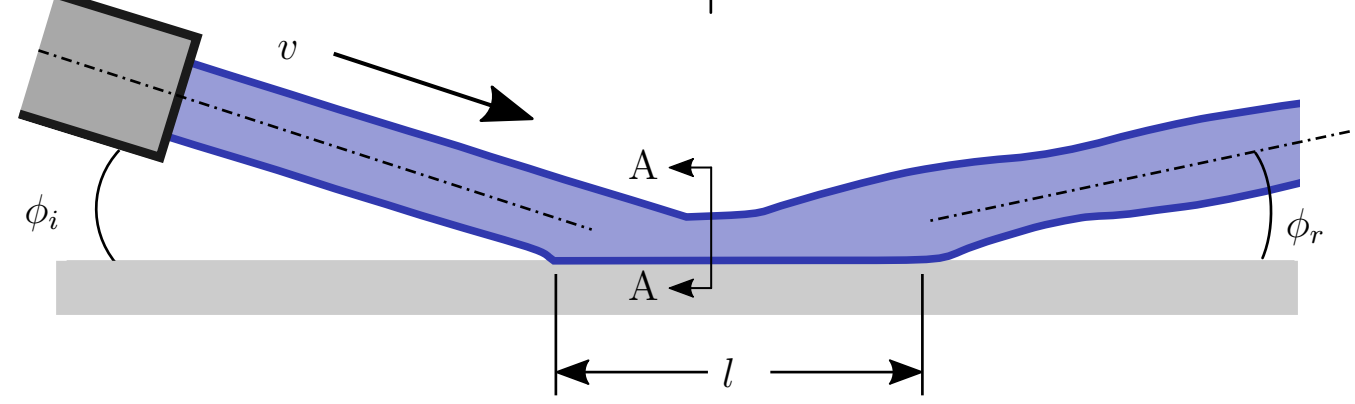

(c)

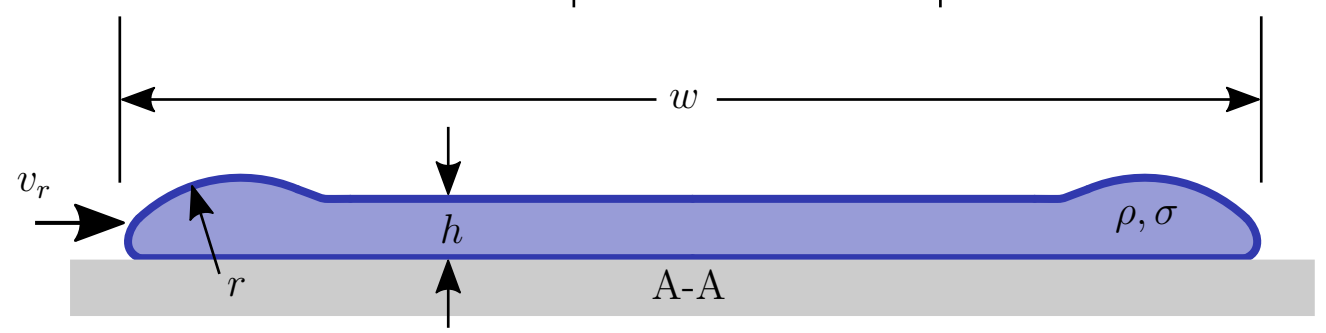

Figure 1.1: Schematic of oblique jet impact with landing flow and rebound from a hydrophobic substrate: (a) top view, (b) profile view, and (c) cross-section view of the landing flow at the point of maximum width $w$ with bounding rims identified by radius $r$.

By employing a drop tower, we expand the parameter space of jet impact by an order of magnitude. This investigation includes 'large' jets with a diameter $d_{j}$ greater than the capillary length $l_{c} \equiv(\sigma / \rho g)^{1 / 2}$. The capillary length is approximately $2.7 \mathrm{~mm}$ for water subjected to terrestrial gravity $g_{o}=9.81 \mathrm{~m} / \mathrm{s}^{2}$. The jet Bond number Bo $\equiv d_{j} / l_{c} \ll 1$ expresses the relative importance of gravity to surface tension and is negligible for the present investigation because free fall drop tower environments routinely achieve brief effective low-gravity levels $g \lesssim 10^{-4} g_{o}$.

As for jet impact on any substrate, jet spreading, splashing, receding, and rebound 
are highly dependent on jet velocity, fluid properties, and incident angle culminating in a perpendicular Weber number $\mathrm{We}_{\perp} \equiv \rho v_{\perp}^{2} d_{j} / \sigma$ and jet Reynolds number $\mathrm{Re} \equiv$ $\rho v d_{j} / \mu$, where $v$ is the jet velocity, $v_{\perp}$ is the jet velocity normal to the surface, $\rho$ is the fluid density, $d_{j}$ is the jet diameter, and $\sigma$ is the surface tension.

Celestini et al. [7] demonstrate jet rebound of submillimetric jets from horizontally oriented hydrophobic substrates with apparent contact angles $\theta^{*}$ of $110^{\circ}$ and $155^{\circ}$. From their data they construct a regime map of stable and unstable rebounds. Celestini et al. [7] also displayed multiple rebounds of a single jet from assemblies of planar hydrophobic substrates. Kibar et al. [15] investigate the spreading area of the landing flow and the force exerted on a variety of vertically oriented hydrophobic substrates by rebounds of 1.75 and $4 \mathrm{~mm}$ diameter jets. Both of these previous investigations focus on low velocity impact regimes where the jet rebounds without splashing.

Jet impingement with superhydrophobic surfaces is dynamically similar to the impingement of two jets. Bush and Hasha [5] investigate the oblique impingement of two jets in low-inertia regimes. At the smallest jet velocities the impact results in a series of mutually orthogonal leaf-shaped chains with thick bounding rims. Each downstream link of such a fluid chain reduces in size until a single deformed jet emerges. As the jet velocity increases the rim destabilizes resulting in a 'fishbone' structure characterized by an organized array of ligaments forming along the sheet edge ultimately creating droplets that eject away from the sheet. Many studies have investigated the impact of two jets at higher velocities $[1,4]$. At high jet velocities ligaments begin to form along the rim, the sheet 'opens' such that there is no re-impingement, and eventually the sheet disintegrates providing a fine spray of droplets. Atomization regimes have received tremendous attention due to their practical application in fuel atomization (e.g., bipropellant rocket engines).

Understanding and controlling jet rebound dynamics, including the landing flow 
structure and secondary jet characteristics, is essential to its application to engineering processes. One of the critical and yet to be determined characteristics of these flows is the transition to a splashing regime. Insight into the landing flow dimensions is also desired. Knowledge of jet rebound mechanics can provide significant contributions to many engineering applications including open-air microfludics, fire suppression on spacecraft, and coating processes.

The drop tower test data collected herein is employed to extend the jet rebound regime map by highlighting landing flow structure as a function of the relevant dimensionless groups Re and $\mathrm{We}_{\perp}$. Building on the work of Celestini et al. [7], we identify new regimes that further subdivide the unstable regime and add novel regimes observed in the limits of large and small impact angles. Simple approximate analytic models are developed for the rim pinching, landing flow residence time, onset of instability, and landing flow dimensions. Following a discussion of substrates, experimental apparatus, procedures, and numerical methods, we present both experimental and computational data for comparisons. The work is concluded with a summary of observations, open questions, and future work. 


\section{Chapter 2}

\section{Theory}

\section{$2.1 \quad$ Wetting}

The wetting characteristics of the substrate are foundational to the description of jet impact, spreading, retraction, and rebound. The most common method to quantify the wettability of a substrate is the optical measurement of the contact angle of a sessile droplet. The Young contact angle $\theta$ describes the contact angle on a smooth chemically homogeneous substrate. For such an ideal substrate, the contact angle depends only on the solid-liquid $(s l)$, solid-gas $(s g)$, and liquid-gas $(l g)$ interfacial tensions. The contact angle may be obtained by balancing these forces acting along the solid plane at the contact line

$$
\sigma_{l g} \cos \theta=\sigma_{s g}-\sigma_{s l}
$$

as shown in Fig. 2.1(a). Equation 2.1 is known as Young's equation. A system is hydrophilic if $0<\theta<90^{\circ}$ and hydrophobic if $90<\theta<180^{\circ}$ as shown in Fig. 2.1. As a point of reference, the largest measured Young contact angle for water known to date is $\theta \approx 120^{\circ}$ for fluorinated substrates [18].

For a flat composite substrate Young's equation is still satisfied along the contact line of the droplet, but the macroscopic, or apparent, contact angle $\theta^{*}$ is defined as 


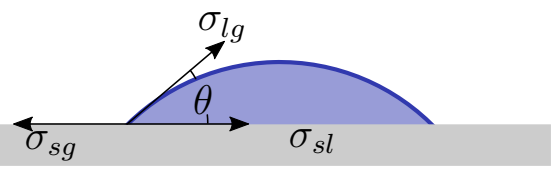

(a)

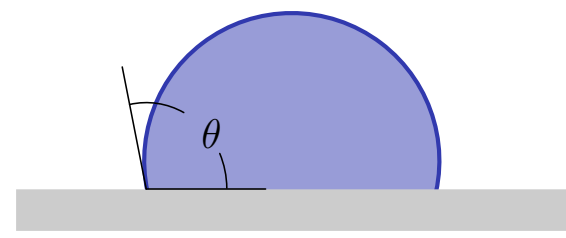

(b)

Figure 2.1: Sessile droplets on flat, chemically uniform hydrophillic (a) and hydrophobic (b) substrate.

an average of the individual contact angles given by

$$
\cos \theta^{*}=\sum_{i}^{n} f_{i} \cos \theta_{i}
$$

where $f_{i}$ is the fraction of the substrate of material $i$ and $\theta_{i}$ is the Young angle for that material. Texturing a hydrophobic substrate can introduce regions of air beneath the droplet, with effective 'contact' angles approaching $180^{\circ}$, increasing the apparent contact angle of the substrate.

Furthermore, substrate roughness alters the contact angle according to Wenzel's Law

$$
\cos \theta^{*}=r_{s} \cos \theta
$$

where $r$ is the ratio of the actual substrate area to its planar projection.

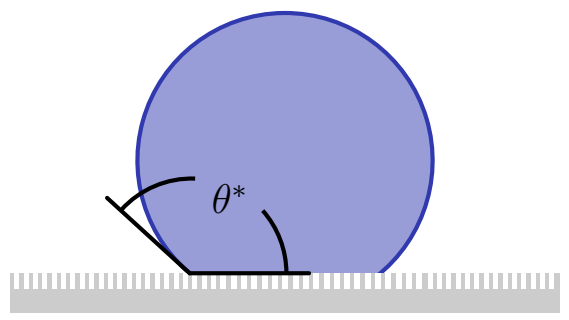

Figure 2.2: A sessile droplet on a textured superhydrophobic substrate.

For a substrate composed of a single material with roughness $r_{s}$, solid fraction 
$f$, and air fraction $(1-f)$ as shown in Fig. 2.2, the apparent contact angle can be expressed as

$$
\cos \theta^{*}=f r_{s} \cos \theta+f-1
$$

Equation 2.4 is a generalization of the Cassie-Baxter equation [6]. In this way textured substrates can achieve superhydrophobic states with $\theta^{*}>150^{\circ}$. Superhydrophobic surfaces provide minimal adhesion of the liquid to the substrate allowing retraction dynamics similar to a fluid surrounded by air. The minimal dissipation provided by the surface during spreading and retraction of the landing flow is essential for jet rebound.

\subsection{Landing Flow Retraction}

During landing flow spreading, a bounding rim is established where the decreasing liquid inertia is resisted and eventually overcome by capillary surface curvature, leading to retraction of the landing flow after the point of maximum width $w$, a collision of the bounding rims, and a rebound of the jet due to the vertical asymmetry of the flow. In other words, the jet prefers the cylindrical state and pushes away from the substrate to maintain that state.

We employ a Taylor-Culick approach commonly used for the inertial retraction of thin films to describe the rim retraction rate $[19,10]$. Considering a sheet with cross section shown in Fig. 1.1(c), a balance between the rate of change of rim momentum $P_{\text {rim }}$ and the surface tension force exerted on the rim yields

$$
\frac{\mathrm{d} P_{\text {rim }}}{\mathrm{d} t}=v_{\text {ret }} \frac{\mathrm{d} m}{\mathrm{~d} t}=2 \sigma,
$$

where $v_{\text {ret }}$ is the constant rim speed and $m$ is the rim mass per unit length. The rate 
of change of the rim mass can be expressed as

$$
\frac{\mathrm{d} m}{\mathrm{~d} t}=\rho h v_{\text {ret }}
$$

Inserting Eq. 2.6 into Eq. 2.5 yields the Taylor-Culick speed

$$
v_{\text {ret }}=\left(\frac{2 \sigma}{\rho h}\right)^{1 / 2}
$$

This retraction velocity can be quantitatively confirmed by considering the convergence angle of the landing flow after the point of maximum width. Using the cross-stream velocity $v_{\text {ret }}$ along with the downstream velocity $v_{\|}$, the convergence angle is estimated by $\beta \approx \tan ^{-1}\left(v_{\text {ret }} / v_{\|}\right)$. This prediction for the landing flow angle shows qualitative agreement with images taken from drop tower tests as shown in Fig. 2.3.

(a)

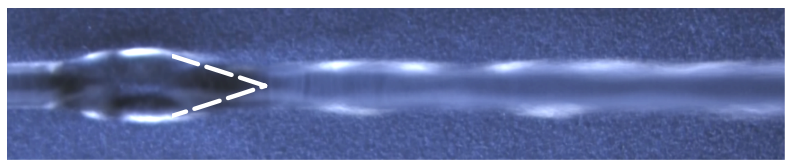

(b)

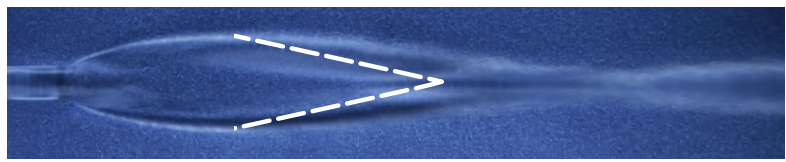

(c)

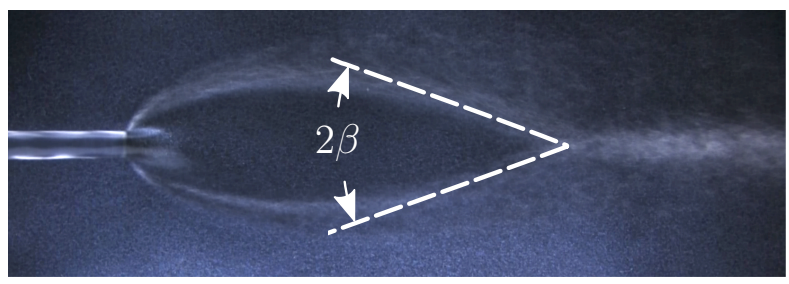

Figure 2.3: Time averaged images from drop tower tests of landing flows with the predicted convergence angle $\beta$ overlaid. Tests: (a) $\phi_{i}=15.1^{\circ}, \mathrm{We}_{\perp}=1.50$ (D1), (b) $\phi_{i}=18.3^{\circ}, \mathrm{We}_{\perp}=10.05$ (D3), and (c) $\phi_{i}=33.5^{\circ}, \mathrm{We}_{\perp}=37.12$ (D4). 
For small $\phi_{i}$, the residence time of the landed jet is largely consumed by such rim retraction towards the apex. Therefore, the landing jet residence time is approximated as

$$
\tau_{r} \sim \frac{w}{2 v_{\mathrm{ret}}} \sim\left(\frac{\rho h w^{2}}{8 \sigma}\right)^{1 / 2}
$$

\subsection{Rim Instability}

For high Weber number impacts, varicose rim perturbations are observed near the point of impact and continue to propagate downstream. Given sufficient time such varicose perturbations lead to the breakup of the bounding rims. The wavelength of the varicose perturbations is close to the most unstable wavelength for a free jet $\lambda \sim 4.5 d_{j}$ [11], which supports the role of the Rayleigh-Plateau instability in the breakup of the rim. Given a rim radius of approximately $2 h$, the relevant time scale for Rayleigh-Plateau pinching and breakup $\tau_{p}$ is the capillary-inertial time scale

$$
\tau_{p} \sim\left(\frac{\rho 8 h^{3}}{\sigma}\right)^{1 / 2}
$$

Later we will compare $\tau_{p}$ to the residence time $\tau_{r}$ to predict the onset of splashing.

\subsection{Energy Model}

It is observed that the value of $w$ depends far more on the jet perpendicular velocity $v_{\perp}$ than tangential jet velocity $v_{\|}$. We conclude that the kinetic energy of the jet normal to the substrate is largely converted to excess landing flow surface energy. Balancing the kinetic and surface energy of the normal velocity free jet and the zero normal velocity landed jet, the landing flow dimensions are estimated $[12,13]$. Before impact the jet is modeled as a cylinder of diameter $d_{j}$ and length $L$ traveling towards the 
substrate at velocity $v_{\perp}$ with the cylinder axis parallel to the substrate. The free jet energy is $E_{1}=E_{K E, 1}+E_{S, 1}$, where $E_{K E, 1}=\frac{1}{8} \rho \pi d_{j}^{2} v_{\perp}^{2} L$ is the initial kinetic energy and $E_{s, 1}=\sigma_{l g} \pi d_{j} L$ is the initial surface energy. The landing flow geometry at maximum width is modeled as a rectangular slab of height $H$, width $W$, and length $L$. The perlength energy of the zero normal velocity landing flow is $E_{2}=(A \sigma)_{s g}+(A \sigma)_{s l}+(A \sigma)_{l g}$ where $A \sigma$ is the surface energy of each solid-liquid $(s l)$, solid-gas $(s g)$, and liquid-gas $(l g)$ interface. Employing the geometric condition $H W=\pi d_{j}^{2} / 4$, the landing flow surface energy may be expressed as $E_{2}=L W\left(r_{s} f\left(\sigma_{s l}-\sigma_{g s}\right)+\left(\frac{\pi d_{j}^{2}}{4 W^{2}}+2-f\right) \sigma_{l g}\right)$. Equating the two energies yields

$$
r_{s} f \frac{\sigma_{g s}-\sigma_{s l}}{\sigma_{l g}}-1+f-\frac{\pi d_{j}^{2}}{2 W^{2}}-1=-\frac{1}{W \sigma_{l g}}\left(\frac{1}{8} \rho \pi d_{j}^{2} v_{\perp}^{2}+\sigma_{l g} \pi d_{j}\right) .
$$

Combining Eq. 2.1 and Eq. 2.4, $r_{s} f \frac{\sigma_{g s}-\sigma_{s l}}{\sigma_{l g}}-1+f=\cos \theta^{*}$. Further simplification to Eq. 2.10 yields a quadratic equation

$$
W^{2} \sigma_{l g}\left(1-\cos \left(\theta^{*}\right)\right)+\sigma_{l g} \frac{\pi d_{j}^{2}}{2}=W\left(\frac{1}{8} \rho \pi d_{j}^{2} v_{\perp}^{2}+\sigma_{l g} \pi d_{j}\right)
$$

The physical, positive root of Eq. 2.11 is

$$
W=\frac{\pi d_{j}}{16} \frac{\left(\mathrm{We}_{\perp}+8\right)}{\left(1-\cos \theta^{*}\right)}\left(1+\left[1-\frac{128\left(1-\cos \theta^{*}\right)}{\pi}\left(\frac{1}{\mathrm{We}_{\perp}+8}\right)^{2}\right]^{1 / 2}\right)
$$

When $\mathrm{We}_{\perp}>>1$, setting $\theta^{*}=180^{\circ}$, solving Eq. 2.12 for the normalized landing flow width $W / d_{j}$ gives

$$
\frac{W}{d_{j}}=C_{1} \frac{\pi\left(\mathrm{We}_{\perp}+8\right)}{16}
$$

where the prefactor $C_{1}$ is introduced as a fit parameter to match experimental data. Equation 2.13 provides a simple prediction for the landing flow dimensions. 


\subsection{Splashing}

As stated, a larger perpendicular Weber number $\mathrm{We}_{\perp}$ increases the time for the Rayleigh-Plateau instability to induce pinching along the landing flow rim. Bulbous regions of the rim draw out ligaments as the rim retracts that eventually pinch off resulting in an array of droplets in the wake of the landing flow. It is expected that the rim will become unstable when the residence time of a fluid parcel in the rim

exceeds the pinching time of the rim: $\tau_{r} / \tau_{p} \gtrsim 1$. Evaluating this criteria with Eq. 2.8 and Eq. 2.9 yields

$$
w\left(\frac{\rho h}{8 \sigma}\right)^{1 / 2}\left(\frac{\sigma}{8 \rho h^{3}}\right)^{1 / 2}=\frac{w}{8 h}>1 .
$$

Letting $h \sim H, w \sim W$, and again using the geometric property $H W=\pi d_{j}^{2} / 4$, Eq. 2.14 becomes

$$
\frac{W}{8} \frac{4 W}{\pi d_{j}^{2}}>1
$$

Instability will occur when

$$
\frac{W}{d_{j}}>(2 \pi)^{1 / 2}
$$

Inserting Eq. 2.13 gives

$$
\mathrm{We}_{\perp}>\frac{16}{C_{1}}\left(\frac{2}{\pi}\right)^{1 / 2}-8,
$$

which provides a critical Weber number for the onset of splashing. 


\section{Chapter 3}

\section{Methodology}

All experiments are performed at the Dryden Drop Tower facility at Portland State University. The Dryden Drop Tower (DDT) is a safe, low-cost, high-rate facility located in the atrium of an engineering building on campus. An image of the tower is provided in Fig. 3.1. During a drop test the rig and drag shield are released simultaneously; because the rig is enclosed in the drag shield, the rig is largely protected from aerodynamic drag during free fall. The drag shield and experiment rig fall $22 \mathrm{~m}$ providing $2.1 \mathrm{~s}$ of relative low-gravity $\mathrm{g}<10^{-4} g_{o}$. Additional DDT introduction details are provided by Wollman [22].

\subsection{Apparatus}

A schematic of the jet rebound experiment rig is sketched in the Fig. 3.2, with devices for fluid injection, jet/substrate positioning, and image capture labeled. An accumulator consisting of two rigid reservoirs separated by a flexible membrane delivers the liquid jets for all tests. The upstream reservoir is filled with air and the downstream reservoir is filled with water. When the solenoid is actuated open, the large upstream reservoir provides ideal gas expansion displacing the membrane and downstream reservoir water through the nozzle at a controlled flow rate. During each $2.1 \mathrm{~s}$ test the gas pressure decreases $<5 \%$ yielding undetectable changes in measurable 


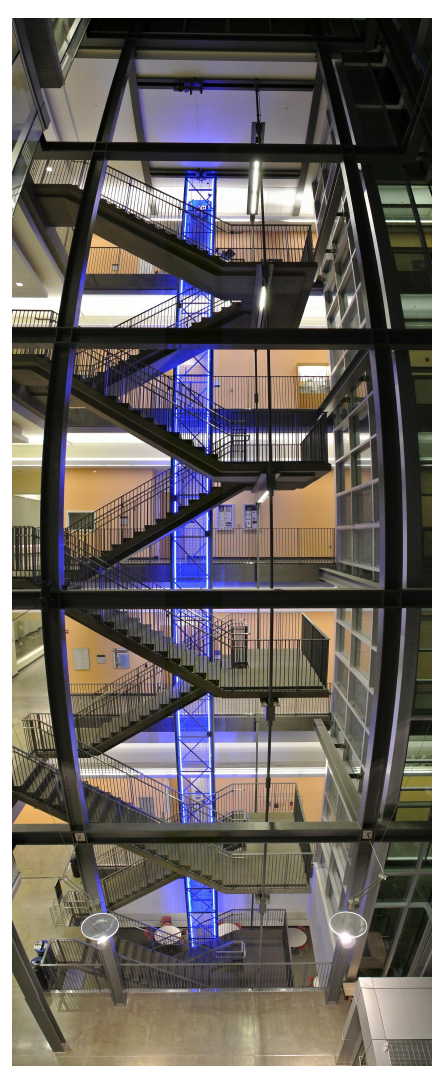

Figure 3.1: Photograph of the Dryden Drop Tower at Portland State University.

jet characteristics such as landing flow length $l$. For each experiment the accumulator is filled with the test fluid followed by air until the desired pressure set point is reached.

The jet steady velocity $v$ is determined via terrestrial calibration using a scale balance and time interval to determine the mean mass flow rate as a function of initial pressure. Jet flow rates $0.1 \leq v \leq 33.8 \mathrm{ml} / \mathrm{s}$ are established. Room temperature water is the working fluid for all tests reported with measured surface tension $\sigma=$ $0.0716 \pm 0.0001 \mathrm{~N} / \mathrm{m}$. The jet nozzles consist of either a blunt commercial stainless steel dispensing needle or a length of stainless steel tubing. Nozzle diameters $d_{j}$ of $0.3,0.5,1.3,1.7,2.4,2.9$, and $6.0 \mathrm{~mm}$ are employed. The nozzles are mounted to a 


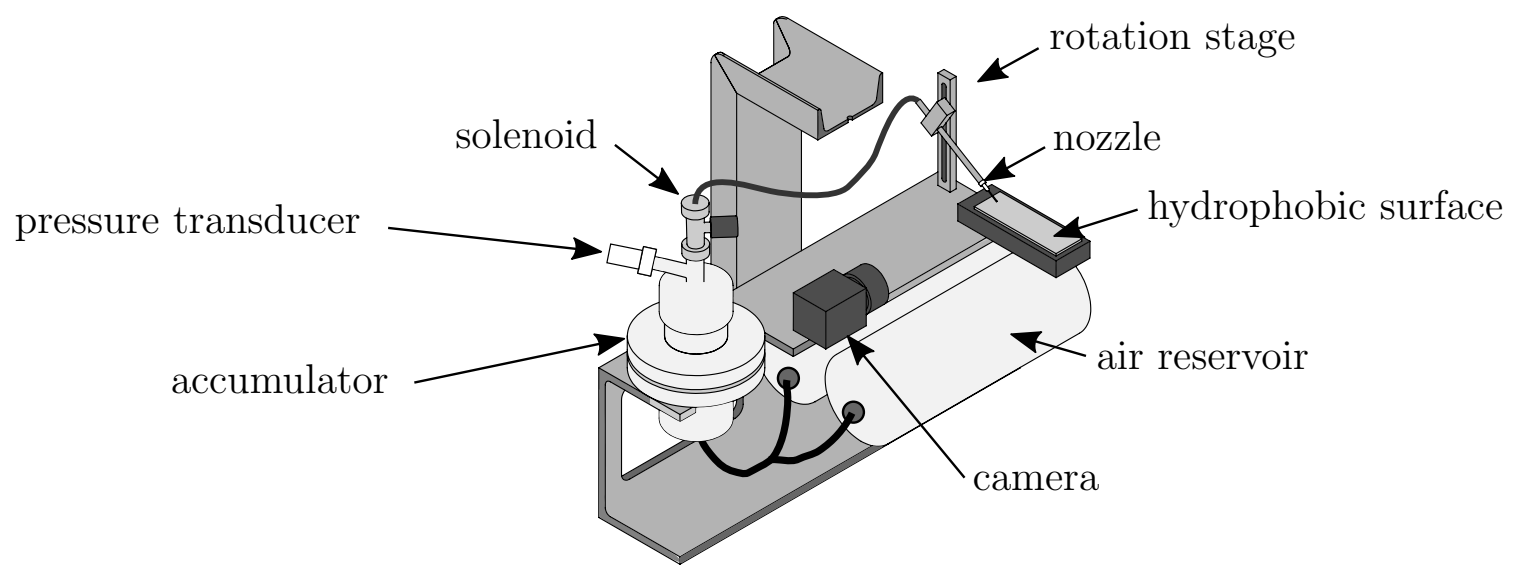

Figure 3.2: Drop tower experiment rig with critical items identified.

manual rotation stage allowing for easy adjustment of the jet-substrate impact angle. A representative set of drop test experiment parameters are included in Table 3.1. A drop index D\# has been assigned to each test case in the table for reference.

\subsection{Hydrophobic Substrate}

A hydrophobic substrate is developed, in part, to support this work. The process creates uniform, repeatable, non-wetting substrates. Our method uses ISO P400 silicon carbide sandpaper bonded to a PMMA plate; the sandpaper provides the surface texture essential for superhydrophobic substrates. The sandpaper surface is then coated with a PTFE aerosol spray (King Controls: Dome Magic) to create a superhydrophobic substrate. The PTFE spray canister is held approximately $0.2 \mathrm{~m}$ above the substrate and sprayed in widthwise strokes with $50 \%$ overlap between adjacent strokes. Each substrate initially receives three applications of PTFE spray with subsequent periodic reapplication between tests. No notable changes in the hydrophobic substrate characteristics are observed during drop tests. The static sessile drop contact angle for water on the substrates measures $\theta^{*}=158 \pm 5^{\circ}$, as determined by the Surface Evolver algorithm [3] via the SE-FIT user interface [8]. 
Table 3.1: Parameters for a representative selection of drop tower tests.

\begin{tabular}{cccccc} 
Drop Index & $d_{j}(\mathrm{~mm})$ & Flow Rate $(\mathrm{mL} / \mathrm{s})$ & $\phi_{i}\left(^{\circ}\right)$ & $\mathrm{We}_{\perp}$ & $w(\mathrm{~mm})$ \\
\hline D1 & 6.0 & 14.40 & 15.1 & 1.50 & 9.14 \\
D2 & 0.5 & 1.00 & 15.7 & 12.53 & 0.74 \\
D3 & 6.0 & 31.00 & 18.3 & 10.05 & 16.18 \\
D4 & 6.0 & 33.80 & 33.5 & 37.12 & 29.16 \\
D5 & 0.5 & 0.80 & 49.3 & 63.23 & 2.84 \\
\hline
\end{tabular}

\subsection{Data Collection and Reduction}

All tests are imaged at 60 or 120 fps with $1920 \times 1080$ pixel resolution from both profile and top perspectives using consumer-grade Panasonic cameras model HC-WX970 or HDC-TM900. Due to limited pixel density, especially for small jet diameters, errors in the landing flow region measurements approach 5\%. A diffuse LED array is adopted to backlight the phenomena. For each test the jet is established before the release of the drop rig into free fall such that both $1-\mathrm{g}$ and low-g data is recorded. The jet rebound regimes are assessed qualitatively from the video records. Quantitative measures of the flow such as landing flow width, impact angle, etc. are extracted using the ImageJ software package [17].

\subsection{Numerical method*}

The numerical approach developed by Wang and Desjardins [20] is employed for benchmark comparisons. The verified level-set computational strategy is accurate, conservative, and robust in simulating inertia-dominated liquid-gas flows with moving contact lines $[20,21]$. The complexity of the jet rebound and the quality of drop tower experiments make for an ideal dataset for further code validation.

*This section was completed in collaboration with Sheng Wang from the Computational ThermoFluids Laboratory at Cornell. 


\section{Chapter 4}

\section{Results}

Following the definitions provided by Celestini et al. [7], 'stable' rebounds are those where the rebounded jet profile in the near impact region is steady with no observed higher harmonic or aperiodic oscillations and no traveling waves, Fig. 4.1(a). Rebounding jets are 'unstable' when unsteady aperiodic oscillations, traveling waves, and premature Rayleigh breakup are observed, Fig. 4.1(b). If the perturbations on the bounding rims are given sufficient time to grow, the rim breaks up and splashing occurs , Fig. 4.1(c). The landing flow structure provides additional distinctions from which to further classify the rebound behavior. The jet rebound regimes shown in Fig. 4.1 are presented in the regime map of Fig. 4.2 in terms of Reynolds number Re and perpendicular Weber number $\mathrm{We}_{\perp}$. All data are collected from the drop tower experiments with symbol size proportional to incident jet diameter.

As observed from the regime map of Fig. 4.2, stable rebounds occur in the lowest inertia laminar region where $\mathrm{Re} \lesssim 3000$ and $\mathrm{We}_{\perp} \lesssim 20$. The stable jet landing flow is characterized by relatively small maximum width $w$ and equally smooth and steady bounding rims. The maximum landing flow width for stable rebounds is less than twice the initial jet diameter. Rim impact asymmetry gives rise to minor-major axis switching oscillations along the rebounded jet. As noted by Celestini et al. [7], the rebounding jet oscillations are similar to those present on jets generated from 

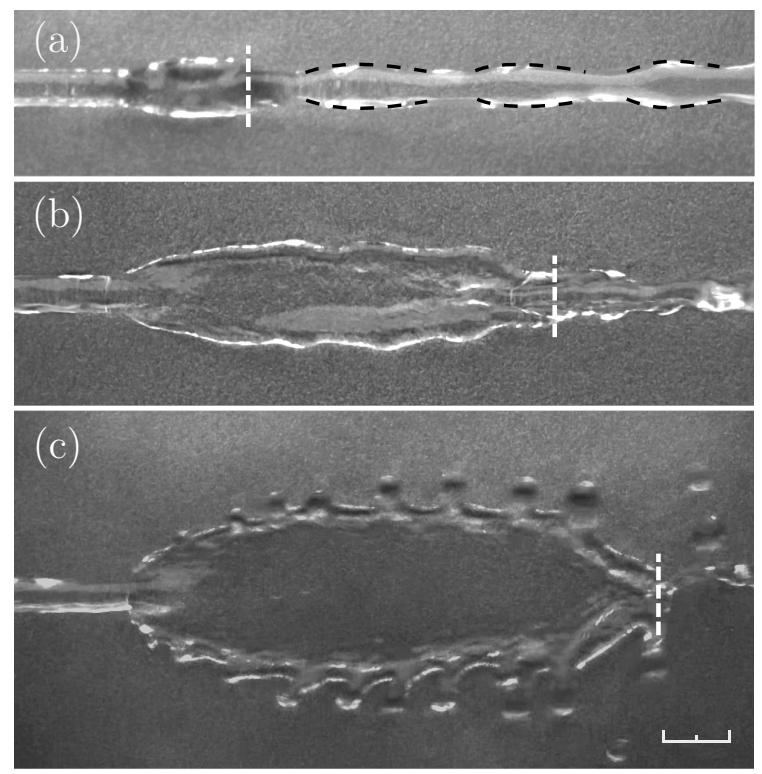

Figure 4.1: Top view still images taken from drop tower test footage of oblique impacts of a water jet with a superhydrophobic $\left(\theta=158^{\circ}\right)$ substrate in a nearly weightless environment. Regimes include: (a) stable, $\phi_{i}=15.1^{\circ}, \mathrm{We}_{\perp}=1.50$ (D1), (b) unstable, $\phi_{i}=18.3^{\circ}, \mathrm{We}_{\perp}=10.05$ (D3), and (c) fishbone, $\phi_{i}=33.5^{\circ}, \mathrm{We}_{\perp}=37.12$ (D4). Light dashed lines indicate the location where the jet leaves the substrate. Dark dashed lines outline the stable rebounded jet profile which does not change with time. Scale bar is $1 \mathrm{~cm}$.

elliptic nozzles. Such oscillations persist until the jet breaks up downstream due to the perturbation growth.

For the low-gravity tests we readily observe stable jet rebounds at low impact angles as the jet incidence becomes parallel to the substrate. Even tangent jets that are sufficiently close to the substrate, where $\phi_{i}=0$ and $\mathrm{We}_{\perp}=0$, are observed to rebound due to the interaction of naturally increasing varicose undulation amplitudes with the substrate, as demonstrated in Fig. 4.3. In a terrestrial environment, such a jet attaches to the substrate as a rivulet as reported by Celestini et al. [7]. However, in low-gravity environments, for such low incident angle impacts $\mathrm{We}_{\perp}$ is not determined by jet velocity but by free jet perturbation growth rates which are essentially normal 


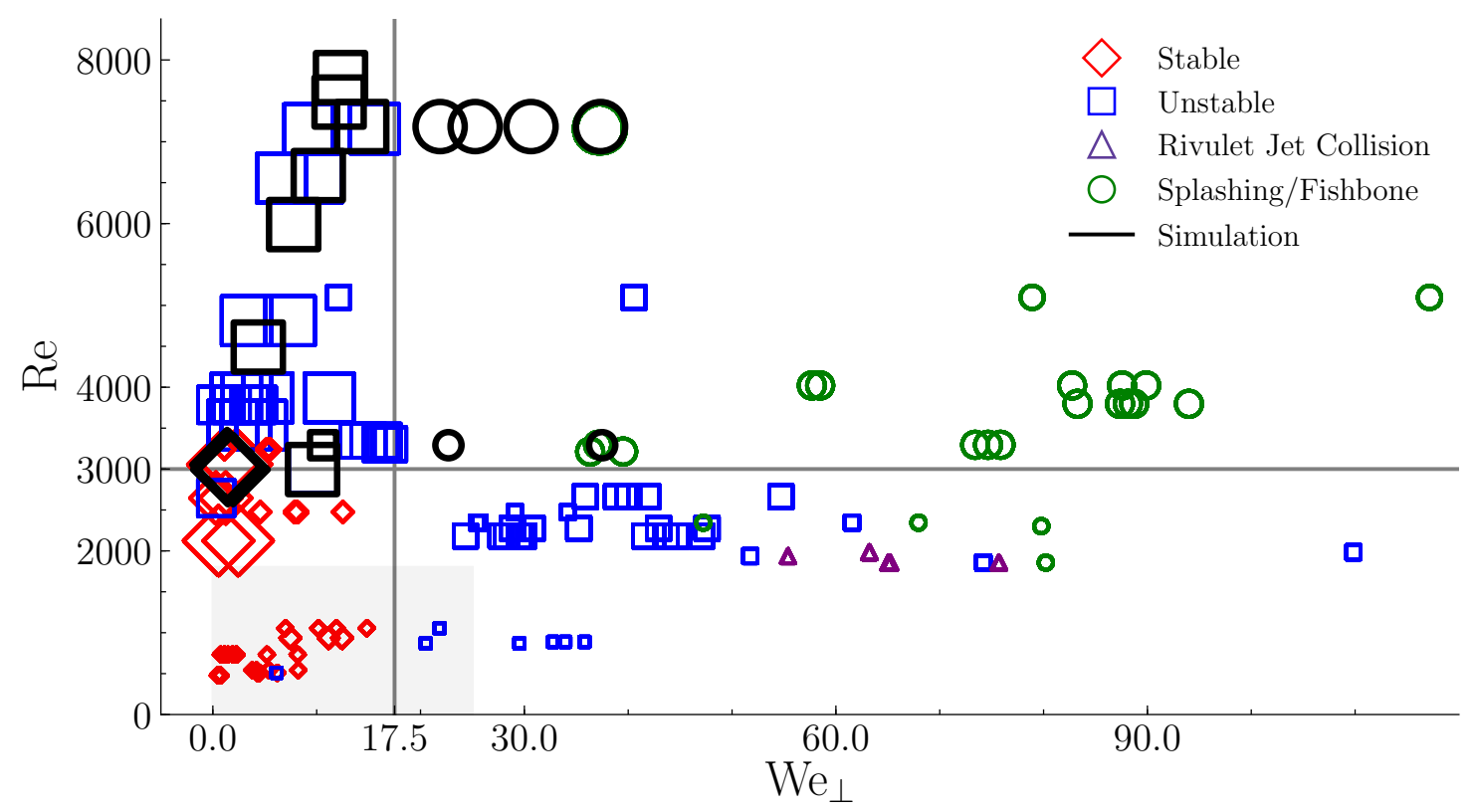

Figure 4.2: Regime map illustrating the observed dependence of flow structures emerging from the oblique impact of a rebounding jet from a superhydrophobic substrate. Marker size is proportional to the diameter of the jet and black bold markers represent numerical simulations. The horizontal line at $\mathrm{Re}=3000$ marks the approximate transition to turbulent flow for the incident jet and the vertical line at $\mathrm{We}_{\perp}=17.5$ marks the predicted onset of splashing behavior (Eq. 2.16 with $C_{1}=1 / 2$. The fit coefficient $C_{1}=1 / 2$ is justified by Fig. 4.4). The shaded region represents the extent of Celestini et al. [7].

to the jet axis. Since such perturbations grow with velocities $v_{p} \sim\left(\sigma / \rho d_{j}\right)^{1 / 2}$, we find an effective perpendicular Weber number, based on the growth velocity $v_{p}$ of $\mathrm{We}_{p} \sim 1$, which lies within the stable rebound regime observed in the experiments when $\operatorname{Re} \lesssim 3000$. If the jet rebounds from the substrate with velocity $v_{p}$, the jet rebound angle can by approximated by

$$
\phi_{r} \sim \frac{180}{\pi} \tan ^{-1}\left[\frac{v_{p}}{v}\right]=\frac{180}{\pi} \tan ^{-1}\left[\left(\frac{1}{\mathrm{We}}\right)^{1 / 2}\right]
$$


Using a small angle expansion Eq. 4.1 yields

$$
\phi_{r} \sim \frac{180}{\pi}\left(\frac{1}{\mathrm{We}}\right)^{1 / 2}
$$

Given the minimum Weber number for jetting of We $\sim 4$ as identified by Clanet and Lasheras [9], from Eq. 4.1 we can expect a maximum rebound angle for a tangent jet of $\phi_{r} \sim 27^{\circ}$.

Outside the stable regime, unsteady varicose perturbations are observed along the free surfaces of the landing flow rims and the rebounded jet. Sources of such perturbations include capillary pinching as well as higher frequency oscillations from fluctuations naturally present in the incoming jet. The perturbations continue beyond the rim impact point and are present on the rebounding jet. Unstable regimes exhibit increased normalized landing flow widths of $1.5 \leq w / d_{j} \leq 9$ as determined using time average composite images of the mean of each pixel for all frames captured during the low-gravity portion of the drop tower tests. The normalized landing flow width ratio $w / d_{j}$ is plotted against $\mathrm{We}_{\perp}$ in Fig. 4.4, where an increasing, somewhat monotonic, dependence is observed.

(a)

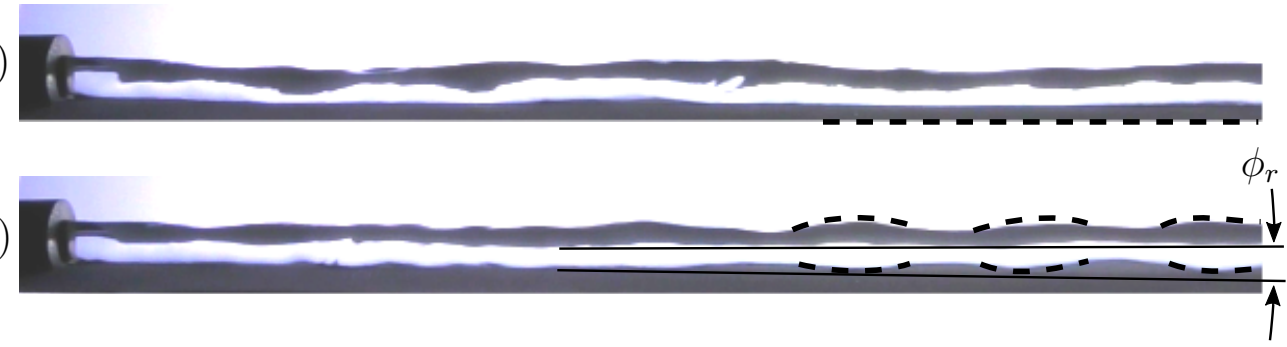

Figure 4.3: Still images of a tangent jet taken from drop tower test footage in (a)1$\mathrm{g}$ before the drop and (b) low-g following the drop package release. In 1-g the jet attaches to the substrate and advances as a rivulet (dashed line). In low-g the growing varicose perturbations deflect the jet away from the substrate (dashed curved segments). The downstream jet in (b) is not in contact with the substrate. 


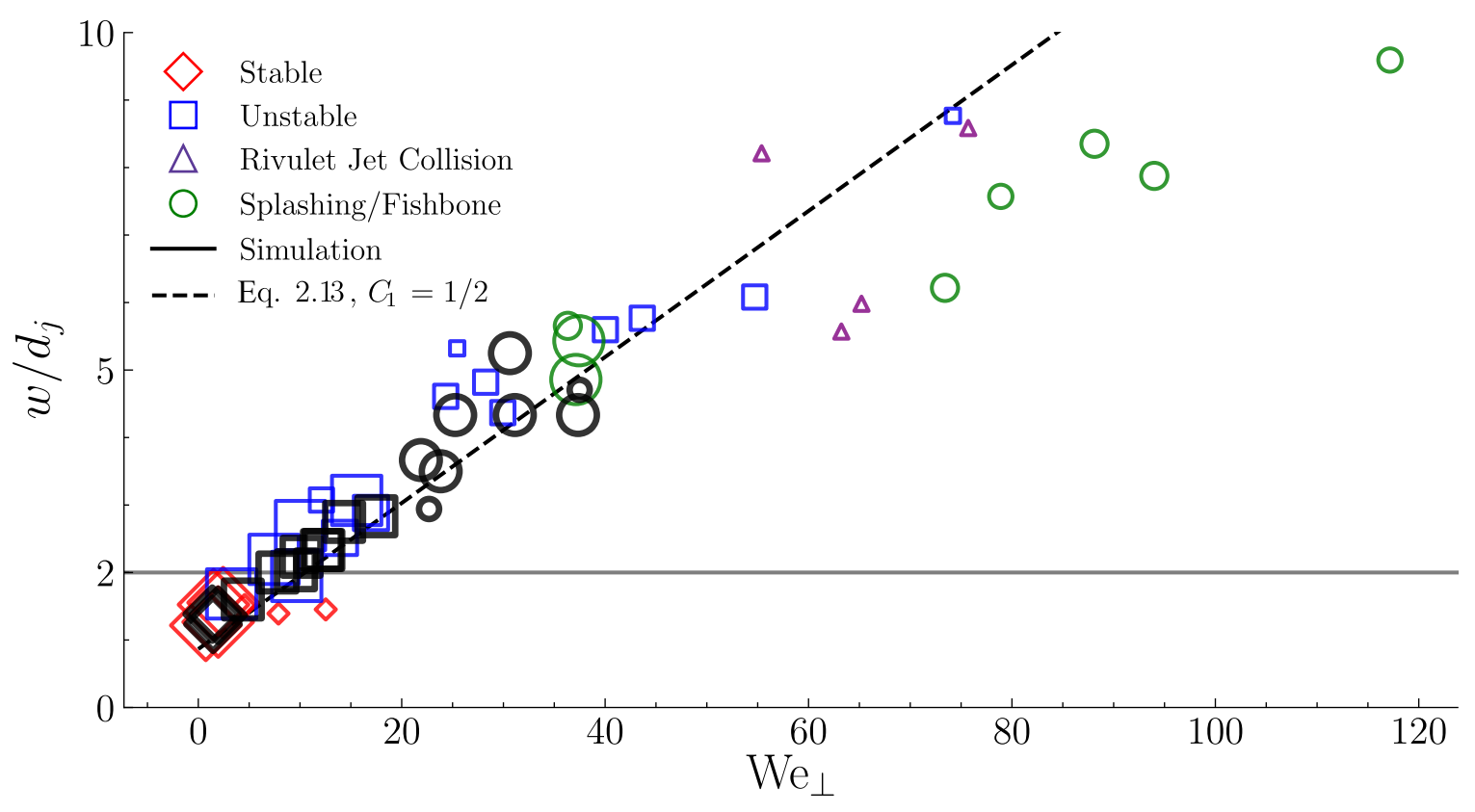

Figure 4.4: Normalized landing flow width $w / d_{j}$ as a function of $\mathrm{We}_{\perp}$. Gray line indicates a maximum normalized landing flow width of $w / d_{j} \sim 2$ for stable rebounds. Marker size is proportional to $d_{j}$ and black bold markers represent simulations.

As $\mathrm{We}_{\perp}$ increases the combination of rim capillary pinching and the radial flow extrudes ligaments that ultimately pinch off as droplets. The droplet formation is periodic and symmetric across the flow centerline. Droplets that detach from the rim also detach from the substrate. Splashing regimes exhibit normalized landing flow widths of $5 \leq w / d_{j} \leq 10$. The landing flow width prediction Eq. 2.13 with $C_{1}=1 / 2$ over-predicts for the splashing regime at high $\mathrm{We}_{\perp}$, Fig. 4.4.

As shown in Fig. 4.2, an 'island' of unique behavior arises for laminar jets when $\mathrm{Re} \approx 2000$ and $8 \lesssim \mathrm{We}_{\perp} \lesssim 50$. In this regime the landing flow forms a terminal third rim at its downstream edge which redirects fluid towards the converging outer rims as shown in Fig. 4.5. A time averaged composite image and sketch of such a landing flow are presented in Fig. 4.5(a). The two rim rivulets and terminal third rim are sketched in Fig. 4.5(b). Both outer rim rivulets rebound from the substrate 
as half-volume jets at the intersection of the primary rivulet rims and the terminal rim. The two rebounding jets then take convergent paths, colliding and coalescing to form a single rebounded jet. The suggestion of this rebound mechanism is supported by the slight rim angle change $\alpha$ at the point of rebound sketched in Fig. 4.5(a).

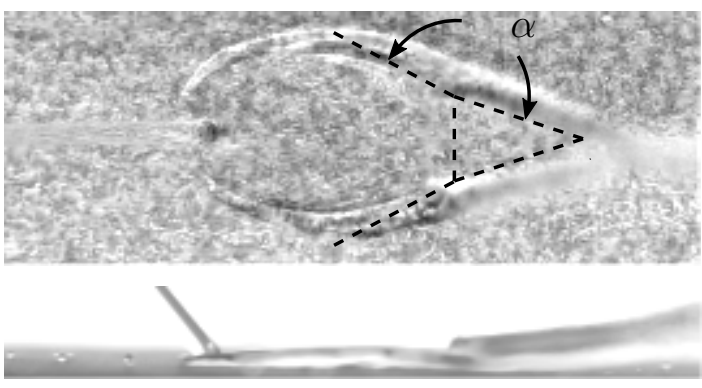

(a)

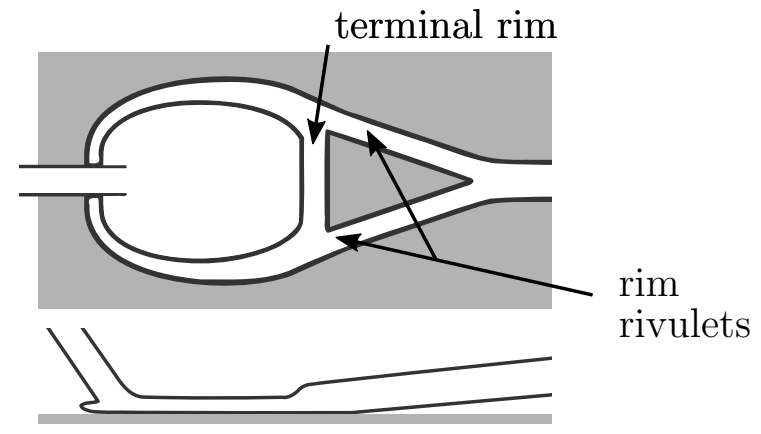

(b)

Figure 4.5: (a) Top and profile views of time-averaged composite image and (b) sketch of the landing region for the double rivulet jet collision regime (D5). The landing flow film splits producing a third 'terminal rim'. The two edge rivulets rebound from the substrate at the point of intersection with the terminal rim only to collide and coalesce downstream forming a single rebounding jet. The triangular void in the flow observed from above is outlined with a dashed line in (a).

As the impact angle approaches a normal impingement, the Weber number of the secondary jet reduces drastically compared to the incoming jet, which results in a flow that may be described more accurately as an attached bulbous blob rather than a jet, as shown in Fig 4.6. Thus, the rebounded jet acts more like 'dripping' flow from a nozzle as opposed to jetting from one. Below a critical Weber number the flow inertia in the secondary jet is not sufficient to induce jetting and may result in periodic large droplet detachments or the growth of a large attached body of fluid. In $1-g_{o}$ the landing flow simply feeds into a large puddle, Fig. 4.6(a); a drastically different outcome from what is observed in low-g.

For the high contact angles and minimal hysteresis in the experiments, the simu- 


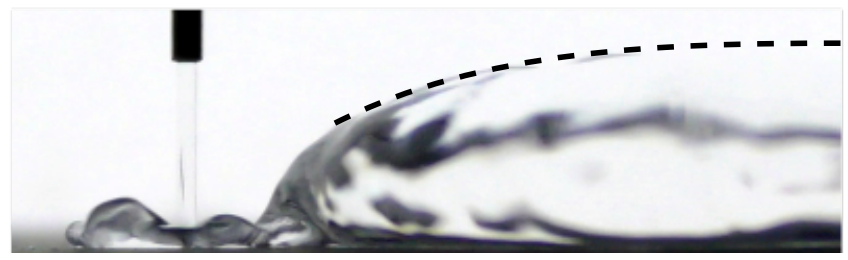

(a)

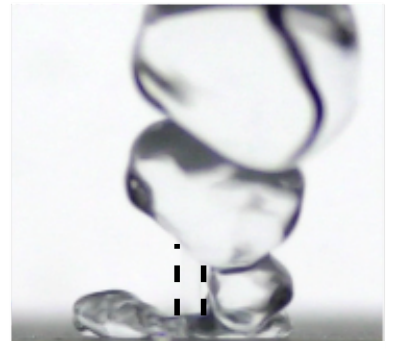

(b)

Figure 4.6: Still images taken from drop tower test footage of a normal jet impingement in 1-g (a) before the drop and low-g (b) after the drop package release. In 1-g the fluid leaving the downstream edge of the landing flow feeds a puddle of height $\sim 2 l_{c}$. In low-g a low Weber number 'jet' is formed which may exhibit dripping behavior, emitting large droplets, and producing a large attached blob. Important features of the fluid free surface are outlined.

lations are able to quantitatively capture features of the landing flow. For both stable and unstable regimes, the qualitative appearance and characteristic landing flow dimensions are captured by the numerical simulations, Fig. 4.7(a,b). Splashing regime simulations capture the obvious flow features of rim destabilization and droplet ejection, Fig. 4.7(c). As shown in Fig. 4.2, simulations agree with the determined regime boundaries. The simulations performed at $\mathrm{Re} \approx 7000$ support the critical weber number of $\mathrm{We}_{\perp} \approx 17.5$. Landing flow widths determined from simulations are included in Fig. 4.4 and show agreement across all regimes. 
(a)
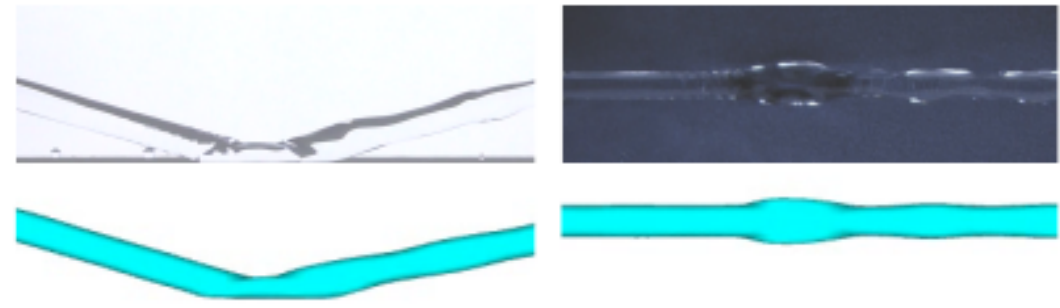

(b)
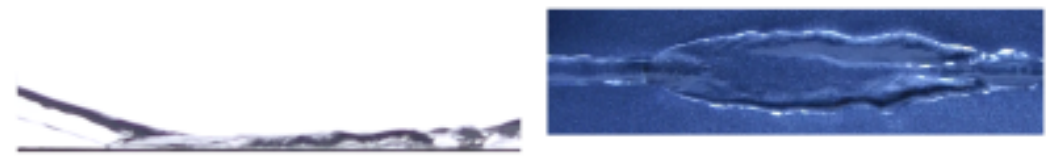

(c)
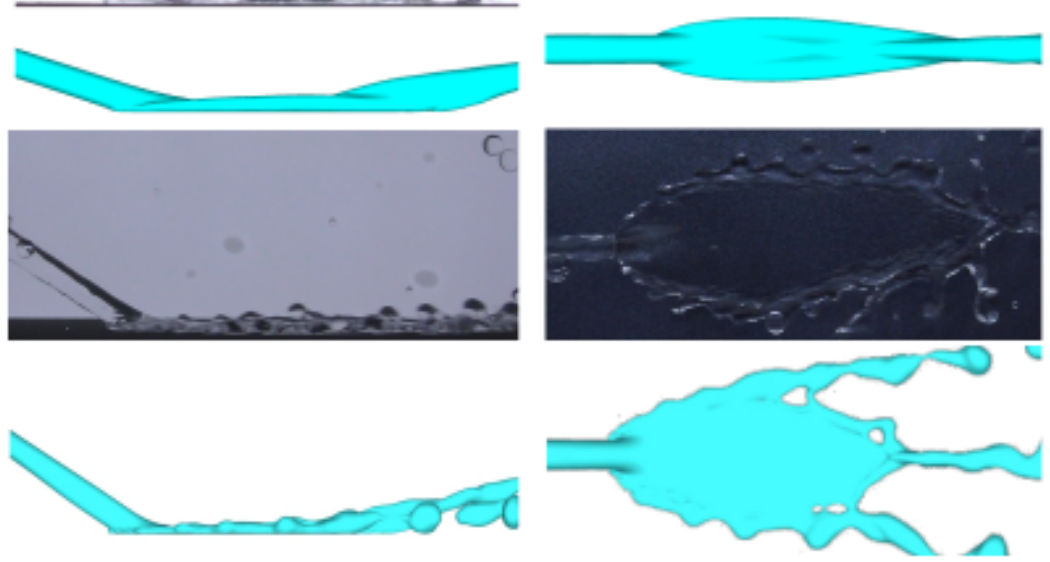

Figure 4.7: Profile and top view comparisons of numerics with drop tower tests for $d_{j}=6 \mathrm{~mm}$ jet. Comparisons include: (a) stable, $\phi_{i}=15.1^{\circ}, \mathrm{We}_{\perp}=1.50$ (D1), (b) unstable, $\phi_{i}=18.3^{\circ}, \mathrm{We}_{\perp}=10.05$ (D3), and (c) fishbone, $\phi_{i}=33.5^{\circ}, \mathrm{We}_{\perp}=37.12$ (D4). 


\section{Chapter 5}

\section{Conclusion}

This work has expanded the regime map for jet rebound by an order of magnitude, spanning the stable and unstable regime, while adding new splashing/fishbone and double rivulet jet collision regimes. The double rivulet jet collision regime has not been previously reported in papers on jet rebound phenomena. We demonstrate that there is no low-g low-angle limit for jet rebound; i.e., even tangent jets rebound. When the impact angle approaches $90^{\circ}$ the rebounded jet enters a dripping regime characterized by intermittent detachment of large droplets or a large fluid body that remains attached to the landing flow.

The energy model we develop predicts the landing flow dimensions as a function of the perpendicular Weber number $\mathrm{We}_{\perp}$. The landing flow width is predicted with an error of $\pm 1.1 d_{j}$. Using the predicted landing flow dimensions in conjunction with models for the rim residence time $\tau_{r}$ and rim capillary pinching time $\tau_{p}$ we determined a critical Weber number $\mathrm{We}_{\perp}$ for splashing. Additional scaling laws are derived for the maximum angle of a tangent jet rebound and the rim convergence angle.

During jet impact the fluid is subjected to an effective acceleration of $a \sim v_{\perp}^{2} / D$. For rebound to occur $\mathrm{Bo}_{a} / \mathrm{Bo}=v_{\perp}^{2} / d_{j} g>1$ where $\mathrm{Bo}_{a}$ is the Bond number based on the acceleration of impact. The transition from the impact shape to a static equilibrium geometry gives rise to the jet rebound. For the jet to rebound we expect 
Bo $\sim 1$; therefore, we expect a minimum perpendicular velocity for terrestrial rebound of $v_{\perp} \sim\left(\sigma / \rho d_{j}\right)^{1 / 2}$, below which rebound will not occur in a terrestrial environment.

Level set numerical simulations capture the landing flow regimes and geometry across the investigated parameter range. The simulations in the splashing region capture the rim destabilization but fail to capture the general flow structure, especially after the point of maximum width. Given the accuracy of the code it would now be prudent to investigating rebounds that cannot be captured in 1-g or drop tower tests.

With a view towards future work, there are many interesting and unaddressed questions remaining. For example, the characteristics of splashing such as the detached droplet size and distribution are left to follow-on studies. Recently, Kibar [14] studied jet rebound from a convex substrate but rebound from a concave substrate remains unstudied. The obvious effect of a concave substrate is that the curvature would act to suppress jet rebound as shown in Fig. 5.1. The additional dissipation associated with viscous fluids and lower contact angles is also a rich direction for investigation. A viscous fluid would slow the growth of perturbations that cause rim pinching and splashing. Near-normal impacts and the transition of the rebounded jet from dripping to jetting could be investigated. Near-normal impacts, viscous impacts, and varying surface wettability could all be supported readily by additional simulations. 


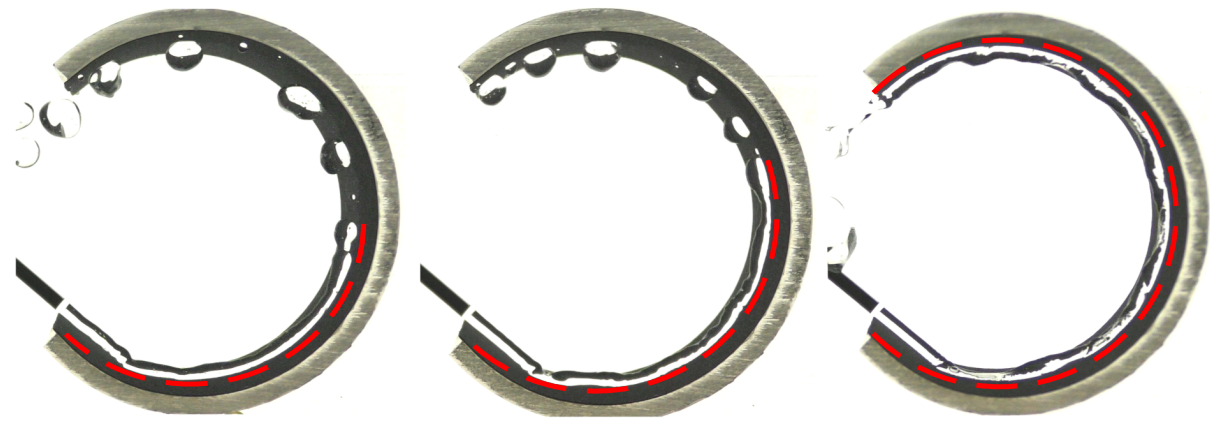

Figure 5.1: Images from drop tower tests showing the impact of a liquid jet with a concave hydrophobic substrate. Flow rate increases from left to right increasing the intact length of the rivulet. The curvature of the substrate suppresses the rebound of the jet. Dashed lines show the rivulet intact length. 


\section{Chapter 6}

\section{Parallel Investigations}

The ease of access to the low-gravity environment that The Dryden Drop Tower facility provides cultivates creativity and discovery. An experimental concept can go from an idea to a drop tower experiment within hours. The following sections encapsulate a selection of peripheral investigations performed at the Dryden Drop Tower Facility during my MSME program.

\subsection{Hazards of Lid Removal in Microgravity}

Due to the potential dangers associated with satellite droplet generation on the International Space Station (ISS), pipetting, mixing, lid removal, and operations essential for Omics are currently performed in the gloveboxes. The characterization and evaluational of the risks associated with such operations are critical as they become more common in the open cabin of the ISS. A drop campaign is performed to provide an example case for demonstrating the potential hazards associated with lid removal from a petri dish in microgravity.

A diagram of the experimental setup is shown in Fig. 6.1(a). The base of petri dish is filled with dyed ethanol and secured to the platform of the drop tower experiment rig. The petri dish lid is attached to a stepper motor shaft by a rigid plastic arm so that the lid is lifted along a circular arc when the motor is energized. The fluid is 
given $0.5 \mathrm{~s}$ to reorient after the step reduction in gravity before the lid is removed to simulate lid removal aboard a spacecraft.

A sequence of images from a drop test are shown in Fig. 6.1(b). A liquid bridge forms between the lid and base of the petri dish. The liquid bridge ruptures creating an explosion of small satellite droplets, Fig 6.1(c). Additional work is currently being performed on lid removal and similar operations such as satellite droplet generation during pipetting. 

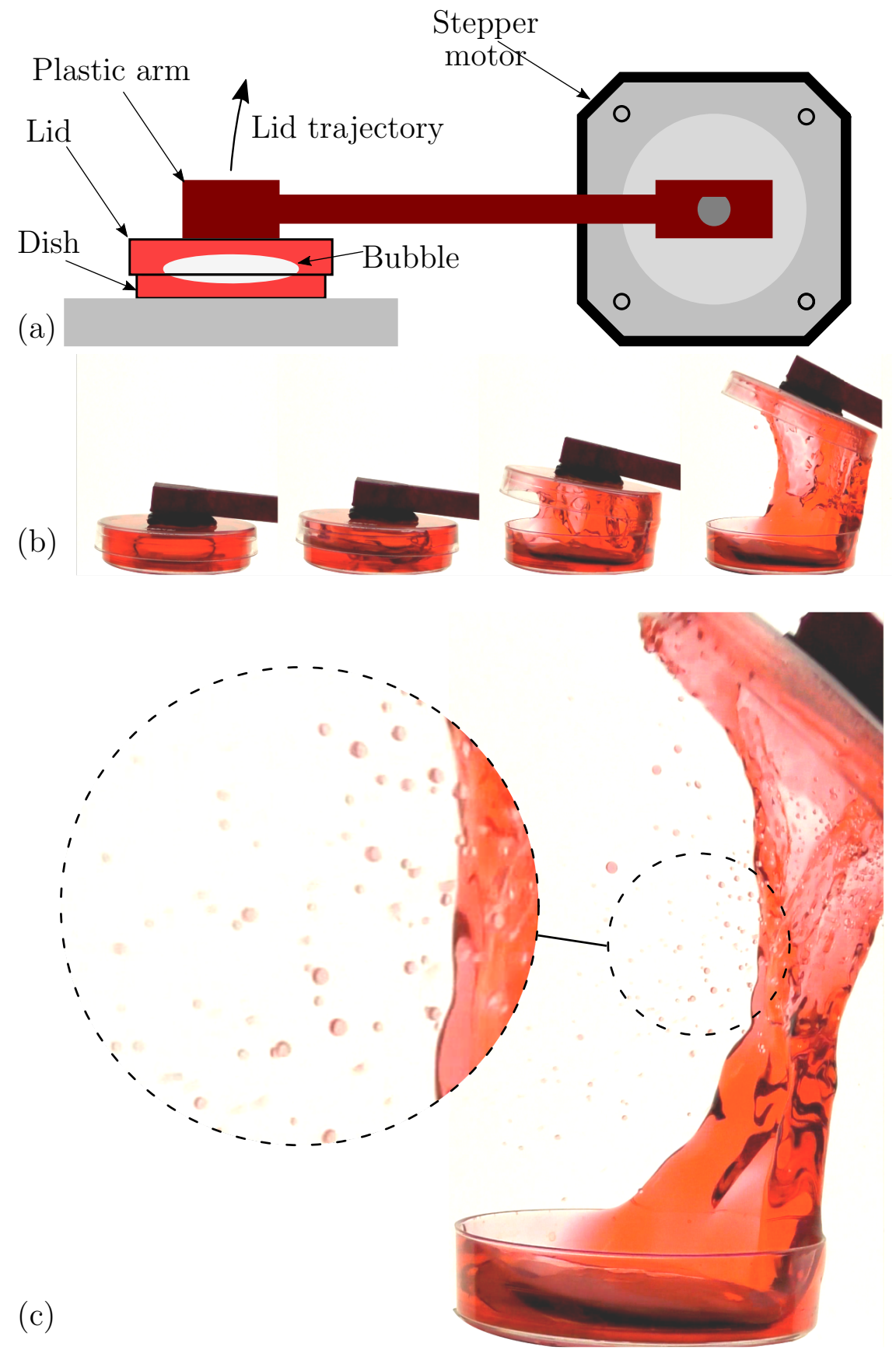

Figure 6.1: (a) A diagram of the experimental setup,(b) an image sequence from lid removal drop tower test showing $\sim 0.5 \mathrm{~s}$ to $2.1 \mathrm{~s}$, and (c) a detail view of the satellite droplets that are generated. 


\subsection{Particle Ejection}

A step reduction in gravity can cause a puddle to reorient and jump from a sufficiently hydrophobic substrate [2]. Small spheres bouncing off the free surface of a liquid has been observed by Lee and Kim [16]. The spontaneous ejection of a hydrophobic particle from a liquid surface in response to a step reduction in gravity, the 'inverse' case of a the puddle jump, is demonstrated here.

A diagram of the experimental setup is shown in Fig. 6.2(a). A $\sim 40 \mathrm{~mm}$ diameter hollow plastic sphere is made hydrophobic by coating it with silicon carbide particles and a PTFE aerosol spray (King Controls: Dome Magic). Before the drop test the sphere is placed in a water reservoir on the drop tower experiment rig. A cup is placed below the sphere to maintain the sphere's position in the middle of the reservoir.

The reorientation of the fluid after the step reduction in gravity causes the sphere to eject, Fig. 6.2(c). Subsequent experiments show that sufficiently non-wetting particles which settle to the bottom of the fluid bath also eject, Fig. 6.2(b).

(a)

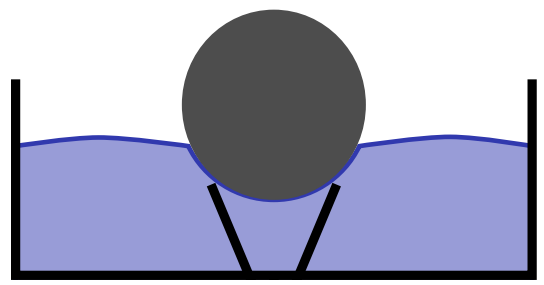

(c)

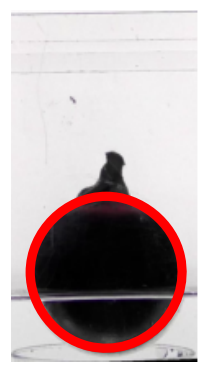

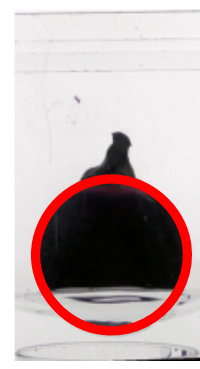

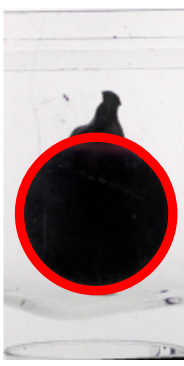

(b)
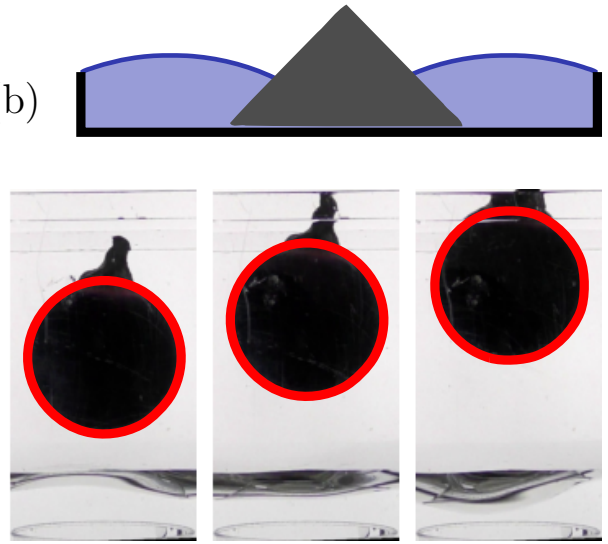

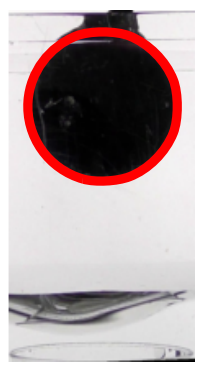

Figure 6.2: Schematic of particle ejection experiment for (a) a buoyant sphere and (b) triangular prism resting on the bottom of the reservoir. (c) Drop tower test image sequence showing the ejection of a $\sim 40 \mathrm{~mm}$ diameter hydrophobic sphere. 


\subsection{Capillary Fluidics Demonstration (Water Ping-Pong)}

The design and characterization of hydrophobic surfaces is a necessary product of the research performed at the DDT lab. A set of ping-pong paddles is designed to be used as an accessible example of the nonintuitive behavior of fluids in low gravity environments. A method of creating a hydrophobic surface from a flight certified material is developed.

We adapt a laser etching process that we had previously used on acrylic to manufacture the paddles from polycarbonate sheet. The laser removes material in a grid leaving a regular array of posts with height $\sim 300 \mu \mathrm{m}$. Spontaneous droplet jump velocity, roll of angle, and static contact angle are compared for a selection of laser power, speed, and pulse frequency to optimize the hydrophobic properties of the paddles.

ESA astronaut Tim Peake used the paddles in a demonstration during an educational outreach event, Fig. 6.3(a), and NASA astronaut Scott Kelly demonstrated the ping-pong paddles on his 300th day in space, 6.3(b). The video of Scott Kelly currently has over one million views on the NASA Johnson YouTube page. I participated in hundreds of drop tower lab tours for elementary to high school aged students and the video of Scott Kelly using the ping-pong paddles often elicits the most audible response. The paddles are also an extension of droplet jump/rebound research [2]. Figure 6.3(c) shows the water droplet trajectory through a sequences of impacts. 


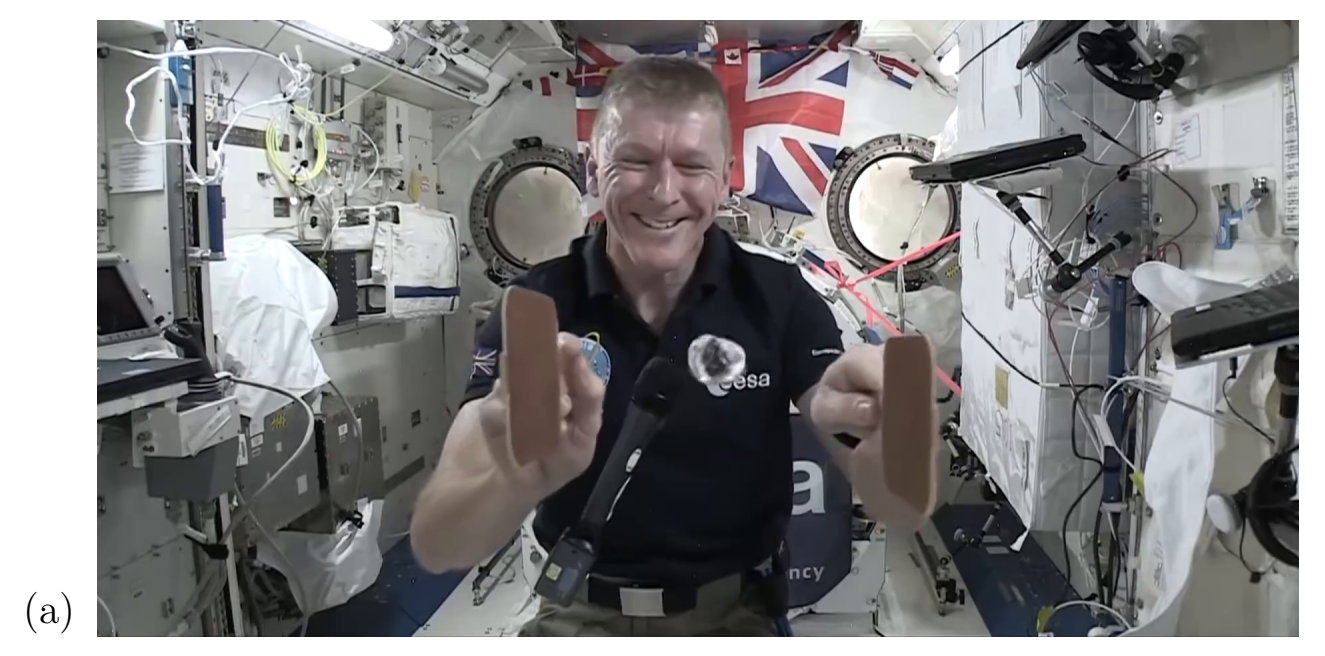

(b)

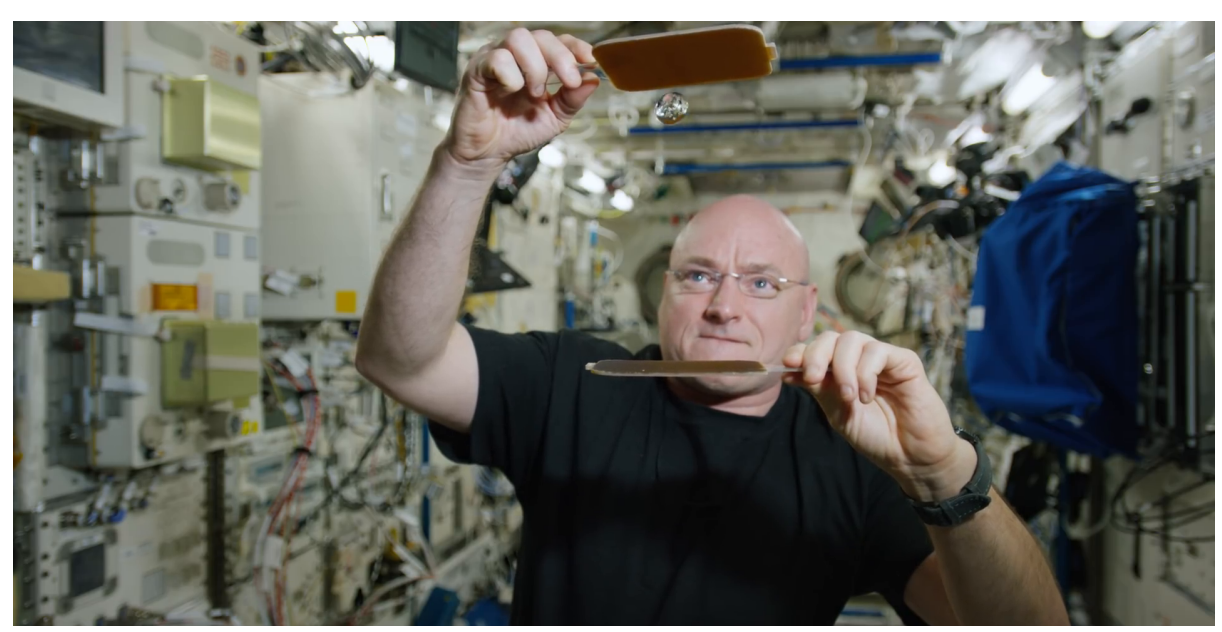

(c)

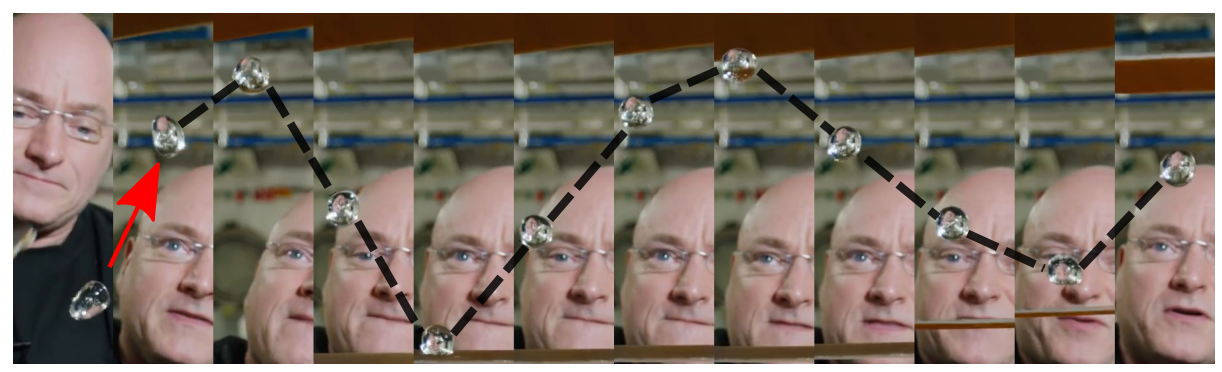

Figure 6.3: (a) ESA astronaut Tim Peake demonstrating the ping-pong paddles to approximately 300 school children during a live stream, (b) NASA astronaut Scott Kelly demonstrating the ping-pong paddles on his 100th day in space, (c) and a time sequence of a droplet bouncing between paddles. 


\section{Bibliography}

[1] Nasser Ashgriz. Handbook of Atomization and Sprays. Springer US, 2011.

[2] B. Attari, M. Weislogel, A. Wollman, Y. Chen, and T. Snyder. Puddle jumping: Spontaneous ejection of large liquid droplets from hydrophobic surfaces during drop tower tests. Physics of Fluids, 28(10):102104, oct 2016.

[3] Kenneth A. Brakke. The surface evolver. Experimental Mathematics, 1(2): 141-165, jan 1992.

[4] N. Bremond and E. Villermaux. Atomization by jet impact. Journal of Fluid Mechanics, 549(-1):273, feb 2006.

[5] Bush W. M. Bush and Alexander E. Hasha. On the collision of laminar jets: fluid chains and fishbones. Journal of Fluid Mechanics, 511:285-310, jul 2004.

[6] A. B. D. Cassie and S. Baxter. Wettability of porous surfaces. Transactions of the Faraday Society, 40:546, 1944.

[7] F. Celestini, R. Kofman, X. Noblin, and M. Pellegrin. Water jet rebounds on hydrophobic surfaces: a first step to jet micro-fluidics. Soft Matter, 6(23):5872, 2010.

[8] Yongkang Chen, Ben Schaffer, Mark Weislogel, and Gregory Zimmerli. Introducing SE-FIT: Surface evolver - fluid interface tool for studying capillary surfaces. 
In 49th AIAA Aerospace Sciences Meeting including the New Horizons Forum and Aerospace Exposition. American Institute of Aeronautics and Astronautics, jan 2011.

[9] Christophe Clanet and Juan C. Lasheras. Transition from dripping to jetting. Journal of Fluid Mechanics, 383:307-326, mar 1999.

[10] F. E. C. Culick. Comments on a ruptured soap film. Journal of Applied Physics, 31(6):1128-1129, jun 1960.

[11] Jens Eggers and Emmanuel Villermaux. Physics of liquid jets. Reports on Progress in Physics, 71(3):036601, feb 2008.

[12] Soeren Kaps, Rainer Adelung, Michael Scharnberg, Franz Faupel, Srdjan Milenkovic, and Achim Walter Hassel. Determining superhydrophobic surfaces from an expanded cassie baxter equation describing simple wettability experiments. arXiv preprint arXiv:1408.5273, 2014.

[13] Ali Kibar. Experimental and numerical investigations of the impingement of an oblique liquid jet onto a superhydrophobic surface: energy transformation. Fluid Dynamics Research, 48(1):015501, dec 2015.

[14] Ali Kibar. Experimental and numerical investigation of liquid jet impingement on superhydrophobic and hydrophobic convex surfaces. Fluid Dynamics Research, 49(1):015502, nov 2016.

[15] Ali Kibar, Hasan Karabay, K. Sleyman Yiğit, Ikrime O. Ucar, and H. Yıldırım Erbil. Experimental investigation of inclined liquid water jet flow onto vertically located superhydrophobic surfaces. Experiments in Fluids, 49(5):1135-1145, apr 2010. 
[16] Duck-Gyu Lee and Ho-Young Kim. Impact of a superhydrophobic sphere onto water. Langmuir, 24(1):142-145, jan 2008.

[17] Johannes Schindelin, Ignacio Arganda-Carreras, Erwin Frise, Verena Kaynig, Mark Longair, Tobias Pietzsch, Stephan Preibisch, Curtis Rueden, Stephan Saalfeld, Benjamin Schmid, Jean-Yves Tinevez, Daniel James White, Volker Hartenstein, Kevin Eliceiri, Pavel Tomancak, and Albert Cardona. Fiji: an opensource platform for biological-image analysis. Nature Methods, 9(7):676-682, jul 2012.

[18] Elaine G. Shafrin and William A. Zisman. Upper limits to the contact angles of liquids on solids. In Advances in Chemistry, pages 145-157. American Chemical Society, jan 1964.

[19] G. Taylor. The dynamics of thin sheets of fluid. III. disintegration of fluid sheets. Proceedings of the Royal Society A: Mathematical, Physical and Engineering Sciences, 253(1274):313-321, dec 1959.

[20] Sheng Wang and Olivier Desjardins. 3D numerical study of large-scale two-phase flows with contact lines and application to drop detachment from a horizontal fiber. International Journal of Multiphase Flow, 101:35-46, 2018.

[21] Sheng Wang and Olivier Desjardins. Numerical study of the critical drop size on a thin horizontal fiber: Effect of fiber shape and contact angle. Chemical Engineering Science, 187:127 - 133, 2018.

[22] Andrew P. Wollman. Capillarity-driven droplet ejection. Master's thesis, Portland State University, 2012. 